\title{
Frequency and Risk Factors of Diabetic Complications among Selected Group of Diabetic Patients: Real-life Scenario from a Developing Country, Bangladesh
}

\author{
Haque HF ${ }^{\mathrm{a}}$, Mitra $\mathrm{P}^{\mathrm{b}}$, Rahim MA ${ }^{\mathrm{c}}$, Afroz Fa ${ }^{\mathrm{a}}$, Afroze $\mathrm{SR}^{\mathrm{a}}$, Ahmed AKMS ${ }^{\mathrm{d}}$, Musa AKM ${ }^{\mathrm{e}}$
}

\begin{abstract}
Background: Diabetes mellitus is a major global public health problem and its complications like coronary artery disease (CAD), stroke, amputations, nephropathy and retinopathy are alarming public health issues. These complications result in significant morbidity, mortality and huge economic burden for the patient and society. This current study aimed to evaluate the frequency of different diabetic complications and their risk factors in a real-life setting.

Methods: This cross-sectional study was done in BIRDEM General Hospital from July to December 2015. Diagnosed adult diabetic patients of either sex irrespective of duration and type of diabetes were consecutively and purposively included in this study. Enrolled patients were evaluated clinically and by laboratory investigation.

Results: Total patients were 400 with female predominance (57.8\%). Mean age was $54.5 \pm 10.9$ years. Majority (57.5\%) were from urban areas, $82 \%$ had hypertension and $37.5 \%$ had dyslipidaemia. Mean duration of diabetes was $11.6 \pm 7.6$ years, mean body mass index was $24.6 \pm 4.5 \mathrm{~kg} / \mathrm{m}^{2}$, mean HbAlc was $9.1 \pm 2.0 \%$. Nephopathy (42.5\%) was the commonest complication followed by retinopathy (37.8\%), neuropathy (29.8\%), $C A D(25.8 \%)$, peripheral vascular disease (14\%) and stroke (11\%). Long duration ( $\geq 5$ yrs) and poor control of diabetes (HbAlc $\geq 7 \%$ ), family history of diabetes, hypertension, dyslipidaemia and male sex were significant risk factors for diabetic complications.

Conclusions: Complications of diabetes are common. Glycaemic control and control of hypertension, dyslipidaemia and weight management may halt or delay the development of complications of diabetes. Patient education is of paramount importance in this regard.
\end{abstract}

Key words: Bangladesh, complication, frequency, diabetes mellitus.

(BIRDEM Med J 2017; 7(2): 143-147)

Author information

a. Dr. Hasna Fahmima Haque, Farhana Afroz, Samira Rahat Afroze, Registrar, Internal Medicine, BIRDEM General Hospital, Dhaka, Bangladesh.

b. Dr. Palash Mitra, Assistant Registrar, Nephrology, BIRDEM General Hospital, Dhaka, Bangladesh.

c. Dr. Muhammad Abdur Rahim, Assistant Professor, Nephrology, BIRDEM General Hospital, Dhaka, Bangladesh.

d. Dr. AKM Shaheen Ahmed, Associate Professor, Internal Medicine, BIRDEM General Hospital, Dhaka, Bangladesh.

e. Prof. AKM Musa, Professor, Internal Medicine, BIRDEM General Hospital, Dhaka, Bangladesh.

Address of correspondence: Dr. Hasna Fahmima Haque, Registrar, Internal Medicine, BIRDEM General Hospital, Dhaka, Bangladesh. Email: drfahmimahaque@gmail.com

Received: October 28, $2016 \quad$ Accepted: February 28, 2017

\section{Introduction}

Diabetes mellitus (DM) is a major global health problem, affecting 415 million people and accounting for 5.0 million deaths in $2015 .^{1-3}$ By 2040 the number of affected people is expected to increase to 642 million globally. ${ }^{1-4}$ Diabetes has become the seventh leading attributable risk factor for burden of disease in South Asian countries. ${ }^{5}$ In the South Asian region, Bangladesh has the second largest number of adults with diabetes (7.1 million adults). ${ }^{1}$ Diabetes is a chronic condition and diabetes-related complications like diabetic neuropathy, nephropathy, retinopathy and diabetic foot ulcer are now alarming public health 
issues. These complications contribute to the decreased quality of life for affected individuals and their families with a devastating long-term effect on their financial and social wellbeing. The complications of DM are far less common and less severe in people who have well controlled blood glucose levels. ${ }^{6,7}$ Diabetes mellitus is associated with 10 to $30 \%$ decrease in life expectancy mainly due to its complications and diabetic patients die at an earlier age than nondiabetics. ${ }^{8}$ The present study was aimed to evaluate the frequency of complications among the selected group of diabetic patients in a tertiary care centre and to identify the risk factors responsible for the complications.

\section{Methods}

This cross-sectional study was done in Bangladesh Institute of Research and Rehabilitation in Diabetes, Endocrine and Metabolic Disorders (BIRDEM) from July to December 2015. Diagnosed adult diabetic patients of either sex, irrespective of duration of diabetes were evaluated in this study. Enrolled patients were evaluated clinically and by laboratory tests. Diabetes due to other cause, gestational diabetes, patients with a past history of neuropathy of another cause and patients with significant hepatic or renal disease were excluded. Body mass index (BMI) was calculated as the weight in kilograms divided by the square of the height in meters. Retinopathy was assessed by experienced ophthalmologists using a direct opthalmoscope with dilated pupils. Peripheral neuropathy was assessed by clinical features, supplemented by monofilament testing. Peripheral vascular disease (PVD) was diagnosed based on clinical symptoms and examination. Nephropathy, stroke and coronary artery disease (CAD) were considered by previous records from diabetic guide book.

\section{Results}

Total patients were 400 with female predominance $(57.8 \%)$. Mean age was $54.5 \pm 10.9$ years. Majority $(57.5 \%)$ were from urban areas, $82 \%$ study population had hypertension and $37.5 \%$ had dyslipidaemia. Baseline characteristics of the study subjects are shown in Table I.
Table I. Base-line characteristics of the study population $(\mathrm{n}=400)$

\begin{tabular}{lc} 
Characteristic & Value \\
\hline Total Patient & 400 \\
Mean age (years) & $55.18 \pm 11.24$ \\
Male: female & $169: 231(1: 1.4)$ \\
Rural: urban:sub urban & $98: 230: 72$ \\
Mean duration of diabetes (years) & $11.6 \pm 7.6$ \\
Mean HbAlc $(\%)$ & $9.1 \pm 2.0$ \\
Mean BMI $\left(\mathrm{kg} / \mathrm{m}^{2}\right)$ & $24.6 \pm 4.5$ \\
\hline
\end{tabular}

Nephopathy (42.5\%) was the commonest complication followed by retinopathy (37.8\%), neuropathy (29.8\%), CAD (25.8\%), PVD (14\%) and stroke (11\%) (Table II).

Table II. Diabetes specific complications among the study population $(\mathrm{n}=400)$

\begin{tabular}{lcc} 
Complication & Frequency & Percentage \\
\hline Microvascular & & \\
Nephropathy & 170 & 42.5 \\
Retinopathy & 151 & 37.8 \\
Neuropathy & 119 & 29.8 \\
Macrovascular & & \\
Coronary artery & 103 & 25.8 \\
disease (CAD) & & \\
Peripheral vascular & 56 & 14 \\
disease (PVD) & & \\
Cerebrovascular & 44 & 11 \\
disease (CVD) & & \\
\hline
\end{tabular}

Duration ( $\geq 5 \mathrm{yrs}$ ) and family history of diabetes, hypertension, dyslipidaemia and male sex were significant risk factors for diabetic complications (Tables III and IV). 
Table III. Risk factors for diabetic microvascular complications ( $\mathrm{n}=400)$

\begin{tabular}{llcccccc} 
Risk factor & & Retinopathy & $\mathrm{p}$ value & Nephro pathy & $\mathrm{p}$ value & Neuropathy & $\mathrm{p}$ value \\
\hline Duration of DM & $<5 \mathrm{yr}$ & 3 & 0.000 & 3 & 0.000 & 11 & 0.002 \\
& $5-10 \mathrm{yr}$ & 37 & & 50 & & 44 & \\
& $>10 \mathrm{yr}$ & 111 & & 117 & & 64 & \\
\multirow{5}{*}{ HbA1c } & $<7$ & 12 & 0.104 & 24 & 0.119 & 12 & 0.730 \\
& 7 or more & 139 & & 146 & & 107 & \\
\multirow{5}{*}{ Dyslipidemia } & Yes & 55 & 0.671 & 59 & 0.281 & 52 & 0.110 \\
& No & 96 & & 111 & & 67 & \\
HTN & Yes & 130 & 0.054 & 154 & 0.000 & 101 & 0.268 \\
& No & 21 & & 16 & & 18 & \\
BMI & $<25$ & 72 & 0.000 & 83 & 0.000 & 76 & 0.325 \\
& 25 or more & 79 & & 87 & & 43 & \\
Sex & Male & 69 & 0.278 & 72 & 0.972 & 61 & 0.020 \\
& Female & 82 & & 98 & & 58 & \\
Family H/O DM & Yes & 75 & 0.678 & 90 & 0.506 & 70 & 0.042 \\
& No & 76 & & 80 & & 49 & \\
\hline
\end{tabular}

Table IV. Risk factors for diabetic macrovascular complications ( $\mathrm{n}=400)$

\begin{tabular}{llcccccc} 
Risk factor & & CAD & p value & Stroke & p value & PVD & p value \\
\hline Duration of DM & $<5$ yr & 17 & 0.520 & 3 & 0.009 & 5 & 0.013 \\
& $5-10 \mathrm{yr}$ & 27 & & 20 & & 24 & \\
& $>10 \mathrm{yr}$ & 59 & & 21 & & 27 & \\
\multirow{3}{*}{ HbA1c } & $<7$ & 6 & 0.043 & 3 & 0.450 & 4 & 0.367 \\
& 7 or more & 97 & & 41 & & 52 & \\
Dyslipidemia & Yes & 43 & 0.333 & 29 & 0.000 & 28 & 0.042 \\
& No & 60 & & 15 & & 28 & \\
HTN & Yes & 83 & 0.841 & 40 & 0.110 & 52 & 0.025 \\
& No & 20 & & 4 & & 4 & \\
BMI & $<25$ & 56 & 0.346 & 22 & 0.365 & 29 & 0.451 \\
& 25 or more & 44 & & 19 & & 24 & \\
Sex & Male & 55 & 0.010 & 27 & 0.007 & 32 & 0.015 \\
& Female & 48 & & 17 & & 24 & \\
Family H/O DM & Yes & 30 & 0.000 & 14 & 0.007 & 23 & 0.115 \\
& No & 73 & & 30 & & 33 & \\
\hline
\end{tabular}




\section{Discussion}

Diabetes mellitus has been recognized as a major public health problem with far reaching consequences, not only for its adverse health impact on individuals, but also for its economic burden on the health care system and society at large. ${ }^{9}$ In this study, we analyzed the classical chronic complications of diabetes.

In our study, among microvascular complications, nephropathy was the most common complication which was not similar to the previous study from our country. ${ }^{10}$ Here we found that, nephropathy was present in $42.5 \%$ of study subjects then retinopathy in $37.8 \%$, neuropathy in $29.8 \%$, CAD in $25.8 \%$, PVD in $14 \%$ and stroke in $11 \%$. But previous study from Bangladesh showed microvascular, macrovascular and severe late complications in $39.2 \%, 9.9 \%$ and $12.1 \%$ of patients respectively. ${ }^{10}$

It was seen from our study that microvascular complications were common among the study subject who had long duration of diabetes, hypertension, obesity and macrovascular complications were common among the patients with male sex, poor glycemic status, dyslipidemia and family history of DM. There are several study from India, ${ }^{11}$ one showed neuropathy was more common (37\%) when compared with retinopathy (17\%) and nephropathy (20\%). Microvascular complications were seen in $48 \%$ of their study population. Increasing age, long duration of diabetes, and higher $\mathrm{HbA} 1 \mathrm{c}$ were the common risk factors for all complications. ${ }^{11}$ Pradeepa $\mathrm{R}$ et al. of India also reported that neuropathy was the most common complication and age, glycated hemoglobin, duration of diabetes, serum triglycerides were responsible for that. ${ }^{12}$ Abougalambou $\mathrm{SS}$ et al. from Malyasia found that the prevalence rate of nephropathy was $91 \%$ followed by neuropathy in $54.7 \%$, retinopathy in $39.3 \%$ and macrovascular complications in $17.5 \%$. The vascular complications were significantly associated with the age $(\mathrm{P}<0.001)$, BMI $(\mathrm{P}<0.001)$, and triglyceride. ${ }^{13}$ Study from Camoroon showed most common complication was neuropathy (40\%) and occurrence of complications was associated with hypertension, duration of diabetes, dyslipidemia, microalbuminuria, 24-hour proteinuria, BMI and HbA1c. ${ }^{14}$ In Ethiopia, chronic complications were present in $58.8 \%$ of diabetic patients. Among those visual disturbance was in $25.68 \%$ and neuropathy in $14.4 \%$ of patients. ${ }^{15}$ Al-Maskari F of United Arab
Emirates found that prevalance of coronary artery disease was in $14.4 \%$, peripheral vascular disease in $11.6 \%$, cerebrovascular disease in $3.5 \%$ and along with diabetes, hypertension and dyslipidemia were the risk factors. ${ }^{16}$

Our study had several limitations. The study period was short and sample size of our study was small. It was a single centered study and data from other centers are required to represent the complications of diabetic patients in Bangladesh. Other limitation was the use of direct ophthalmoscopy instead of retinal photography in the assessment of diabetic retinopathy and for detection of neuropathy we did only clinical examination, we could do nerve conduction study and for PVD duplex study of lower limb vessels.

So, it can be concluded like that among the diabetic patients the frequency of complications were high. Increased occurrence of peripheral neuropathy, nephropathy and retinopathy were observed with longer duration of illness. Poor glycemic status, dyslipidemia, hypertension, obesity were the major risk factor along with DM. Diabetic patients need more efforts to be spent on them. Screening and intervention programs should be implemented early at the diagnosis stage and risk factors should be treated aggressively. If we can improve the current status of diabetic patients not only from tertiary care hospital but also from primary care centre, we hope to decrease the rate of frequency of vascular complications.

Acknowledgement: We express our acknowledgement to all our colleagues who have collected data from the study subjects for the study purpose.

Conflict of interest: None declared.

Declaration: This paper was presented in the 33rd World Congress of Internal Medicine, Bali, Indonesia, 2016.

\section{References}

1. International Diabetes Federation. IDF Diabetes Atlas, 7 ed. Brussels, Belgium: International Diabetes Federation, 2015.

2. Danaei G, Finucane MM, Lu Y, Lu Y, Singh GM, Cowan MJ, et al. National, regional and global trends in fasting plasma glucose and diabetes prevalence since 1980: systematic analysis of health examination surveys and epidemiological studies with 370 country-years and 27 million participants. Lancet 2011; 378: 31-40.

3. Mendis S, Puska P, Norrving B, World Health Organization, World Heart Federation, World Stroke Organization. Global 
atlas on cardiovascular disease prevention and control. Geneva: World Health Organization; 2011.

4. Guariguata L, Whiting D, Hambleton I, Beagley J, Linnenkamp U, Shaw J. Global estimates of diabetes prevalence for 2013 and projections for 2035 for the IDF Diabetes Atlas. Diabetes Res Clin Pract 2013; 100(2): 137-49.

5. Lim SS, Vos T, Flaxman AD, Danaei G, Shibuya K, AdairRohani $\mathrm{H}$, et al. A comparative risk assessment of burden of disease and injury attributable to 67 risk factors and risk factor clusters in 21 regions, 1990-2010: a systematic analysis for the Global Burden of Disease Study 2010. Lancet 2013; 380: 2224-60.

6. Nathan DM, Cleary PA, Backlund JY, Genuth SM, Lachin $\mathrm{JM}$, Orchard TJ, et al. Intensive diabetes treatment and cardiovascular disease in patients with type 1 diabetes. N Engl J Med 2005;353(25):2643- 53.

7. The effect of intensive diabetes therapy on the development and progression of neuropathy. The Diabetes Control and Complications Trial Research Group. Ann Intern Med 1995; 122(8):561-68.

8. Buse JB, Ginsberg HN, Bakris GL, Clark NG, Costa F, Eckel $\mathrm{R}$, et al. Primary prevention of cardiovascular diseases in people with diabetes mellitus: a scientific statement from the American Heart Association and the American Diabetes Association. Diabetes Care 2007;30(6):162-72.

9. Heisler M, Pletu JD, Spencer M, Kieffer E, Vijay S. The relationship between knowledge of recent $\mathrm{HbA} / \mathrm{c}$ values and diabetes care undertstanding and self management. Diabetes care 2005; 28:816-22.

10. Latif ZA, Jain A, Rahman MM.Evaluation of management, control, complications and psychosocial aspects of diabetics in Bangladesh: DiabCare Bangladesh 2008. Bangladesh Med Res Counc Bull 2011 Apr;37(1):11-16

11. Kumar HKVS, Kota SK, Basile A, Modi KD.Profile of Microvascular Disease in Type 2 Diabetes in a Tertiary Health Care Hospital in India.Ann Med Health Sci Res 2012; 2(2): 103-108.

12. Pradeepa R, Anjana RM, Unnikrishnan R, Ganesan A, Mohan $\mathrm{V}$ and Rema M. Diabetes Technology \& Therapeutics 2010; 12(10): 755-61.

13. Abougalambou SS, Mohamed M, Sulaiman SA, Abougalambou SA, Hassali MA .Current clinical status and complications among type 2 diabetic patients in University Sains Malaysia hospital. International Journal of Diabetes Mellitus 2010 (2):184-88.

14. Tamba SM, EwaneME, BonnyA, MuisiCN, NanaE, EllongA et al. Pan African Medical Journal 2013; 15:141.

15. Abejew AA, Belay ZA, Kerie MW. Diabetic complication among adult diabetic patients of a tertiary hospital in North east Ethiopia. Hindawi Publishing Corporation Advances in Public Health ;Volume 2015, Article ID 290920, 7 pages. (accessed January 7, 2017).

16. Maskari FA, Sadig ME, Norman JN. The prevalence of macrovascular complications among diabetic patients in the United Arab Emirates Cardiovascular Diabetology 2007; 6: 24. 